\title{
The N1-suppression effect for self-initiated sounds is independent of attention
}

\author{
Jana Timm*, Iria SanMiguel, Katja Saupe and Erich Schröger
}

\begin{abstract}
Background: If we initiate a sound by our own motor behavior, the N1 component of the auditory event-related brain potential (ERP) that the sound elicits is attenuated compared to the N1 elicited by the same sound when it is initiated externally. It has been suggested that this N1 suppression results from an internal predictive mechanism that is in the service of discriminating the sensory consequences of one's own actions from other sensory input. As the N1-suppression effect is becoming a popular approach to investigate predictive processing in cognitive and social neuroscience, it is important to exclude an alternative interpretation not related to prediction. According to the attentional account, the $\mathrm{N} 1$ suppression is due to a difference in the allocation of attention between self- and externally-initiated sounds. To test this hypothesis, we manipulated the allocation of attention to the sounds in different blocks: Attention was directed either to the sounds, to the own motor acts or to visual stimuli. If attention causes the N1-suppression effect, then manipulating attention should affect the effect for self-initiated sounds.

Results: We found N1 suppression in all conditions. The N1 per se was affected by attention, but there was no interaction between attention and self-initiation effects. This implies that self-initiation N1 effects are not caused by attention.

Conclusions: The present results support the assumption that the N1-suppression effect for self-initiated sounds indicates the operation of an internal predictive mechanism. Furthermore, while attention had an influence on the $\mathrm{N} 1 \mathrm{a}, \mathrm{N} 1 \mathrm{~b}$, and N1c components, the N1-suppression effect was confined to the N1b and N1c subcomponents suggesting that the major contribution to the auditory N1-suppression effect is circumscribed to late N1 components.
\end{abstract}

Keywords: Event-related brain potentials, Sensory suppression, Auditory N1 component, Attention, Predictive processing

\section{Background}

It is important to differentiate sensory information resulting from one's own actions from environmental events which are not the result of our own actions. It has been proposed that this differentiation is based on an internal forward model [1-3], an idea that relates to the reafference principle [4] and the concept of corollary discharge [5] in physiological literature. Specifically, when a movement is executed, a copy of the current motor command (efference copy) is used to make predictions of the sensory consequences of the movement (corollary discharge). This sensory prediction is then compared with the actual sensory feedback. If the

\footnotetext{
* Correspondence: jana.timm@uni-leipzig.de

Institute of Psychology, University of Leipzig, Seeburgstr. 14-20, Leipzig D-04103, Germany
}

(c) 2013 Timm et al.; licensee BioMed Central Ltd. This is an Open Access article distributed under the terms of the Creative Commons Attribution License (http://creativecommons.org/licenses/by/2.0), which permits unrestricted use, distribution, and reproduction in any medium, provided the original work is properly cited. two correspond, sensory responses are attenuated, thereby enabling a differentiation between the sensory consequences of one's own actions and the actions of others. Such sensory attenuation for self-generated compared to externally-generated sensations - as an index of an internal predictive mechanism - has been widely investigated in psychophysical research [6,7].

Within this self-generation framework, the N1 suppression paradigm has become a popular approach to investigate predictive auditory sensory processing [8-14]. In this paradigm, participants listen to sounds that are either initiated by their own button presses, or externally initiated. The N1 component of the event-related brain potential (ERP) is attenuated for the sounds that were selfinitiated compared to the externally-initiated sounds. This N1-suppression effect has been explained as the result of 
an underlying predictive mechanism. In the traditional blocked version of this paradigm, self-initiated sounds and externally-initiated sounds are presented in different blocks, bearing several caveats that obscure an unambiguous interpretation in terms of the predictive coding framework [15]. For example, it seems possible that the participants' arousal level differs between the active condition in which participants initiate the sound by their own motor behavior and the passive condition in which participants simply listen to the externally-initiated sounds. In a modified so-called mixed N1 suppression paradigm selfinitiated and externally-initiated sounds are presented within the same block. Thus, sustained arousal differences between self- and externally-initiated sounds are eliminated. Studies using this paradigm also yielded (an even larger) N1-suppression effect for self-initiated sounds $[16,17]$. This demonstrates that the N1-suppression effect seems to occur selectively for self-initiated sounds and seems not to be caused by different arousal levels in active and passive conditions of the blocked design.

Although sustained differences in arousal are well controlled in this mixed design, it is obvious that transient arousal effects cannot be controlled for. Even more important, the improved paradigm has not been designed for excluding attentional influences on the N1-suppression effect. In fact, an enlarged P3a to externally-initiated sounds compared to the P3a for self-initiated sounds reported for the mixed design [16] suggests that externally-initiated sounds received more attention. As the N1 is known to increase with attention [18-22], it seems well possible that differences in the N1 between self- and externally-initiated sounds were in fact caused by a difference in attention directed to self- and externally-initiated sounds. The cognitive psychologist's silver bullet to test for an attentional confound on an effect of interest (here, the N1suppression effect) is to vary the allocation of attention over several levels and determine its influence on the effect $[23,24]$. Therefore, we measured the N1-suppression effect with the mixed design ${ }^{\mathrm{a}}$ and manipulated the allocation of attention between blocks comprising three different attention conditions: While participants are performing the selfinitiation task, attention is directed either to the sounds, the motor acts or to visual stimuli. Less attention should be directed to the sounds when participants attend to the motor act or to the visual stimuli than when they attend to the sounds. If the N1-suppression effect critically depends on an attentional difference, no (or a reduced) N1 suppression should occur when equating attention to externally and self-initiated sounds. In contrast, if N1 suppression for self-initiated sounds reflects a genuine suppression effect rather than an attentional difference, we expect comparable N1 suppression in all three attention conditions, supporting the assumption of an underlying genuine internal predictive mechanism.
Moreover, in order to focus on effects that truly reflect attenuation of sensory responses due to a match of incoming stimulation with predicted stimulation in sensory cortex, we will make a more detailed analysis of the auditory N1, separating suppression effects for the N1a, N1b, and N1c components [25,26]. It is well known that sensory and non-sensory (unspecific) components contribute to the auditory N1 [25]. Importantly, only sensory components with sources in auditory cortex are tangentially oriented, showing a fronto-central distribution with polarity inversion at the mastoids. Contrary, the unspecific component, which reflects the orienting response, appears slightly later in time than tangential components and shows no polarity reversal at the mastoids, as it does not originate in auditory cortex. If the N1-suppression effect truly reflects attenuation of sensory responses that match internal sensory predictions, then sensory-specific components generated in auditory cortex should be attenuated. If on the contrary the N1-suppression effect mostly reflects differences in the orienting response generated by self- and externallyinitiated sounds then the unspecific N1 component should be most affected. Finally, by comparing the N1-suppression effects due to self-initiation and the N1attention effects, we can determine whether the predictive modeling (putatively) underlying the N1 suppression resembles attention effects. Indeed, previous research has reported attention in time effects that share characteristics of attention to other feature effects [20,27].

\section{Results}

Behavioral data

Table 1 summarizes the behavioral results for the selfinitiation task (inter-press time intervals, total number of button presses, timing errors) and the attention task (counting rates) obtained in the three attention conditions $(A S, A M, A V)$. For the self-initiation task the analysis revealed no main effect of Attention for inter-press time intervals $[F(2,24)=0.29 ; p=.749]$, total number of button presses $[F(2,24)=2.31 ; p=.120]$ and timing errors

Table 1 Behavioral results for all three attention conditions (AS, AM, AV)

\begin{tabular}{lccc}
\hline & $\begin{array}{c}\text { Attention } \\
\text { Sounds }(\boldsymbol{A S})\end{array}$ & $\begin{array}{c}\text { Attention } \\
\text { Motor }(\boldsymbol{A M})\end{array}$ & $\begin{array}{c}\text { Attention } \\
\text { Visual }(\boldsymbol{A V})\end{array}$ \\
\hline $\begin{array}{l}\text { Self-initiation task } \\
\begin{array}{l}\text { Interval button } \\
\text { presses (ms) }\end{array}\end{array}$ & $6233(386)$ & $6153(425)$ & $6188(359)$ \\
$\begin{array}{l}\text { Number of button } \\
\text { presses }\end{array}$ & $29.23(2.8)$ & $31.30(2.5)$ & $29.38(2.78)$ \\
$\begin{array}{l}\text { Timing errors (\%) } \\
\text { Attention task }\end{array}$ & $2.33(4.16)$ & $2.94(3.91)$ & $1.06(2.88)$ \\
Counting rates (\%) & $98.37(4.59)$ & $99.34(3.71)$ & $97.24(3.59)$ \\
\hline
\end{tabular}

SD is given in parentheses. 
$[F(2,24)=0.80 ; p=.457]$. However, with regard to the attention task a main effect of Attention was observed $[F(2,24)=5.22 ; p<.05]$. Pairwise comparisons showed lower counting rates for the $A M$ condition compared to the $A V$ condition $[t(12)=4.22 ; p=.001]$. However, the effect size of this effect is low $\left(\mathrm{n}^{2}=0.30\right)$. No differences were obtained comparing $A S$ to $A M[t(12)=-1.43$; $p=.176]$ or $A S$ to $A V[t(12)=1.50 ; p=.158]$. Taken together, no fundamental differences of task demands were observed between the three attention conditions.

\section{Electrophysiological data}

In Figure 1A the grand-average auditory response across all conditions is depicted at central, temporal and mastoid electrodes. The ERP waveform shows a negative deflection in the typical N1 latency range at $\mathrm{Cz}$ and a double-peaked N1 at temporal electrodes with polarity inversion at the mastoids for only the early peak. Voltage maps and scalp current densities (Figure 1B) show the corresponding distributions for this deflection over the scalp in the N1b (85-150 ms), the N1a (60-100 ms) and the N1c (115-150 ms) time window, respectively. In the following, modulations of this auditory response caused by self-initiation and attention are reported. Statistical results for all time windows are presented in Table 2. Most importantly, for all three N1 time windows no interaction of the experimental factors Production and Attention was found (N1b window: $F(2,24)=0.85$; $p=.407$; N1a window: $F(2,24)=6.24 ; p=.536$; N1c window: $F(2,24)=0.80 ; p=.430)$. Thus, auditory $\mathrm{N} 1$ effects due to self-initiation and due to the allocation of attention for each time window will be presented separately (see Additional file 1 for grand-average ERPs as well as voltage maps and scalp current densities (SCDs) of single attention conditions $A S, A M, A V$ ). Furthermore, no interaction of Attention $\times$ Production was observed for the analysis of the mastoids $[F(2,24)=0.72 ; p=$ .495]. Thus, effects due to attention and self-initiation will be discussed separately as well.

\section{Self-initiation effects on the auditory N1}

In Figure 2 grand-average ERP waveforms at $\mathrm{Cz}$ elicited by externally-initiated sounds and self-initiated sounds as well as the self-initiation effect (externally-initiated minus self-initiated) are shown, separately for the three attention conditions. Since comparable self-initiation effects were obtained in all attention conditions ( $A S$, $A M, A V)$ the mean of all three attention conditions was

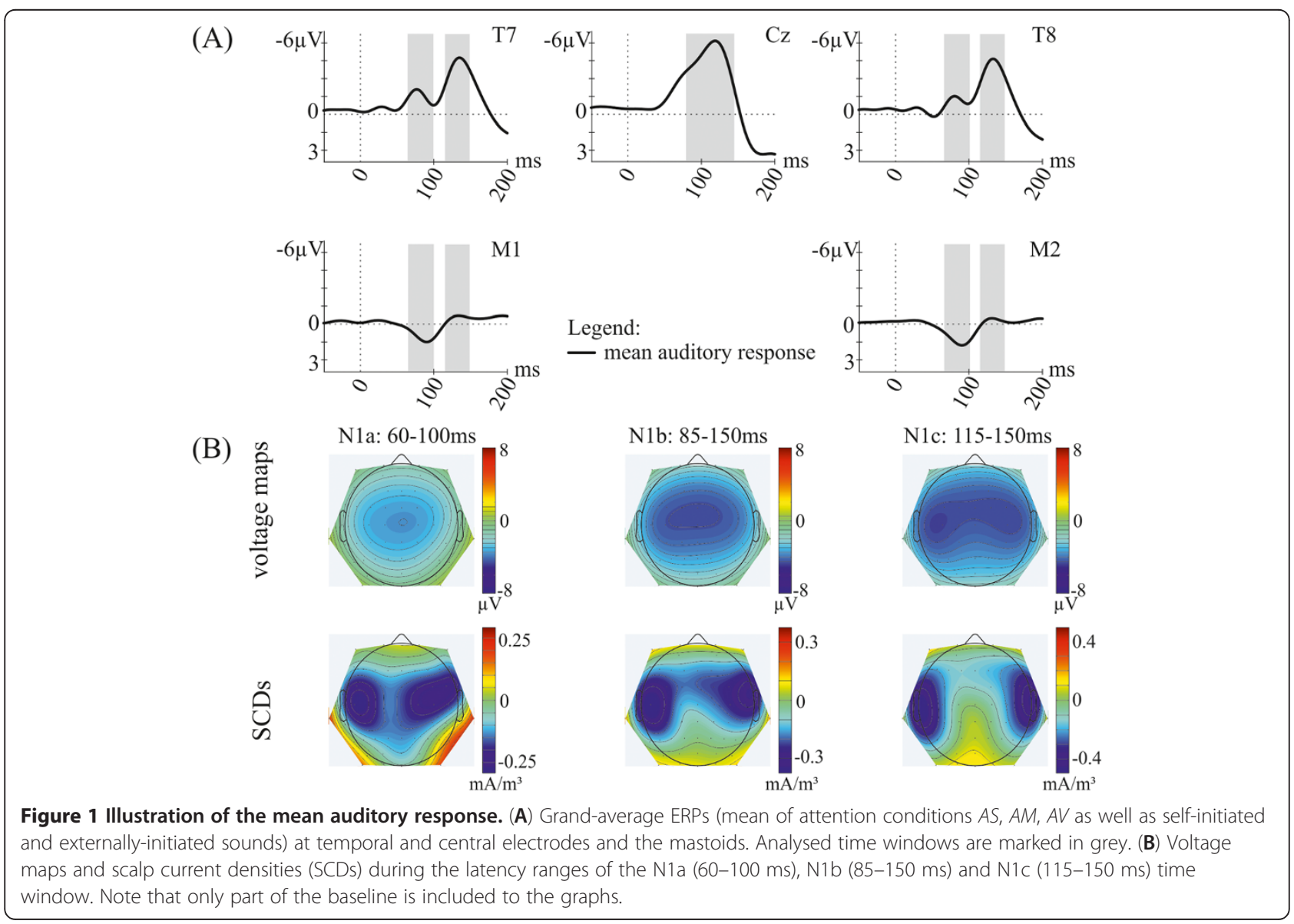


Table 2 Results of the ANOVA for all N1 time windows

\begin{tabular}{|c|c|c|c|c|c|c|c|c|c|}
\hline \multirow{2}{*}{ Attention ${ }^{2}$} & \multicolumn{3}{|c|}{$\begin{array}{l}\text { N1b time window } \\
(85-150 \mathrm{~ms})\end{array}$} & \multicolumn{3}{|c|}{$\begin{array}{l}\text { N1a time window } \\
(60-100 \mathrm{~ms})\end{array}$} & \multicolumn{3}{|c|}{$\begin{array}{l}\text { N1c time window } \\
(115-150 \mathrm{~ms})\end{array}$} \\
\hline & $\begin{array}{l}\boldsymbol{F} \\
32.45\end{array}$ & $p_{* *}^{p}$ & $\begin{array}{l}\mathbf{n}^{2} \\
.730\end{array}$ & $\begin{array}{l}\boldsymbol{F} \\
10.57\end{array}$ & $\begin{array}{l}p \\
* *\end{array}$ & $\begin{array}{l}\mathrm{n}^{2} \\
.468\end{array}$ & $\begin{array}{l}\boldsymbol{F} \\
38.39\end{array}$ & $p_{* *}^{p}$ & $\begin{array}{l}\mathrm{n}^{2} \\
.762\end{array}$ \\
\hline Production $^{1}$ & 18.31 & ** & .604 & 1.61 & .228 & .118 & 24.95 & $* *$ & .675 \\
\hline Laterality $^{3}$ & 38.46 & $* *$ & .762 & 36.71 & * & .754 & 10.37 & $* *$ & .464 \\
\hline AnteriorPosterior $^{2}$ & 6.32 & $*$ & .345 & 7.96 & $* *$ & .339 & 3.80 & .062 & .241 \\
\hline Attention $\mathrm{x}$ Production ${ }^{2}$ & 0.85 & .407 & .066 & 6.24 & .536 & .049 & 0.80 & .430 & .063 \\
\hline Attention $\times$ Laterality ${ }^{4}$ & 9.65 & $* *$ & .446 & 4.82 & * & .287 & 9.93 & $* *$ & .453 \\
\hline Attention $\times$ AnteriorPosterior ${ }^{3}$ & 17.83 & $* *$ & .598 & 6.37 & * & .347 & 9.02 & $* *$ & .434 \\
\hline Production $x$ Laterality $^{3}$ & 30.85 & $* *$ & .720 & 3.02 & .076 & .201 & 11.97 & $* *$ & .499 \\
\hline Production $\times$ AnteriorPosterior ${ }^{2}$ & 4.05 & .058 & .253 & 2.90 & .093 & .194 & 3.76 & .071 & .239 \\
\hline Laterality $\times$ AnteriorPosterior ${ }^{4}$ & 2.03 & .123 & .145 & 4.71 & * & .282 & 2.91 & * & .196 \\
\hline Attention $\times$ Laterality $\times$ AnteriorPosterior $^{5}$ & 1.69 & .155 & .123 & 1.70 & .150 & .124 & 2.53 & * & .174 \\
\hline Production $\times$ Laterality $\times$ AnteriorPosterior ${ }^{4}$ & 2.85 & * & .192 & 2.61 & .051 & .179 & 1.74 & .168 & .127 \\
\hline Attention $\times$ Production $\times$ Laterality ${ }^{4}$ & 1.92 & .148 & .138 & 1.12 & .349 & .085 & 1.56 & .215 & .115 \\
\hline Attention $\times$ Production $\times$ AnteriorPosterior ${ }^{3}$ & 0.66 & .548 & .052 & 0.53 & .618 & .042 & 0.43 & .657 & .034 \\
\hline Attention $\times$ Production $\times$ Laterality $\times$ AnteriorPosterior ${ }^{5}$ & 1.15 & .340 & .088 & 1.18 & .324 & .090 & 1.24 & .295 & .094 \\
\hline
\end{tabular}

$F$ values, $p$ values and partial $\eta^{2}$ for each $\mathrm{N} 1$ time window are reported.

${ }^{1} F(1,12)$.

${ }^{2} F(2,24)$.

${ }^{3} F(4,48)$.

${ }^{4} F(8,96)$.

${ }^{5} F(16,192)$

** $p \leq .001$

$* p \leq .05$.

calculated and used for the further analysis. Figure 3A shows the grand-average ERP waveforms at $\mathrm{Cz}$ for the mean of all three attention conditions ( $A S, A M, A V)$ elicited by externally-initiated sounds and self-initiated sounds as well as the self-initiation effect (externallyinitiated minus self-initiated). Furthermore, voltage maps and scalp current densities (SCDs) show the corresponding distribution over the scalp of the mean self-initiation effect in all three N1 time windows (Figure 3B). The analysis for the N1b time window revealed a main effect of Production $[F(1,12)=18.31 ; p=.001]$. Also for the N1c time window a significant main effect $[F(1,12)=24.95$; $p<.001]$ was observed. This main effect of Production for both time windows was caused by lower amplitudes for self-initiated sounds compared to externally-initiated sounds. However, for the N1a time window no main effect of Production was found $[F(1,12)=1.61 ; p=.228]$, showing comparable amplitudes for self-initiated and externally-initiated sounds. Furthermore, for the N1b time window an interaction of Production $\times$ Laterality $\times$ Anterior-Posterior $[F(8,96)=2.85 ; p=.039]$ was obtained. Pairwise comparisons revealed lower amplitudes at frontal and central electrodes $(p<.05$ for F3, F4, Fz, F7, F8, C3, $\mathrm{Cz}, \mathrm{C} 4)$ for self-initiated compared to externally-initiated sounds, indicating a fronto-central distribution of the selfinitiation effect (see Figure 3B, upper panel). The SCD topography of this effect also shows a pattern pointing at a fronto-central effect (see Figure 3B, lower panel). For the N1c time window no such interaction was observed $[F(8,96)=1.74 ; p=.168]$. However, the analysis revealed an interaction of Production $\times$ Laterality $[F(4,48)=11.97$; $p=.001]$, showing a more central than lateral distribution of the self-initiation effect (see Figure 3B, upper panel). Again, the SCD distribution supports a fronto-central effect (see Figure 3B, lower panel). Contrary, for the N1a time window no interaction with the experimental factor Production was found. Additionally, at the mastoids no main effect of Production was obtained $[F(1,12)=2.98$; $p=.110]$.

\section{Comparison of self-initiation and attention effects on the auditory N1}

In the following, attention effects are outlined and then compared to the self-initiation effect. In order to simplify the comparison, we focused on effects of attending (AS) vs. not attending (AM, AV) to sounds, pooling the attention effects for the AM and AV conditions, which were rather similar (cf. Additional file 2). Thus, we compared effects of attending to sounds (AS vs. $[\mathrm{AM}+\mathrm{AV}] / 2$ [over all production conditions]) to effects of self-initiating the sounds (A-MA [over all attention conditions]). Figure 4A shows the grand-average ERP waveforms at $\mathrm{Cz}$ elicited 

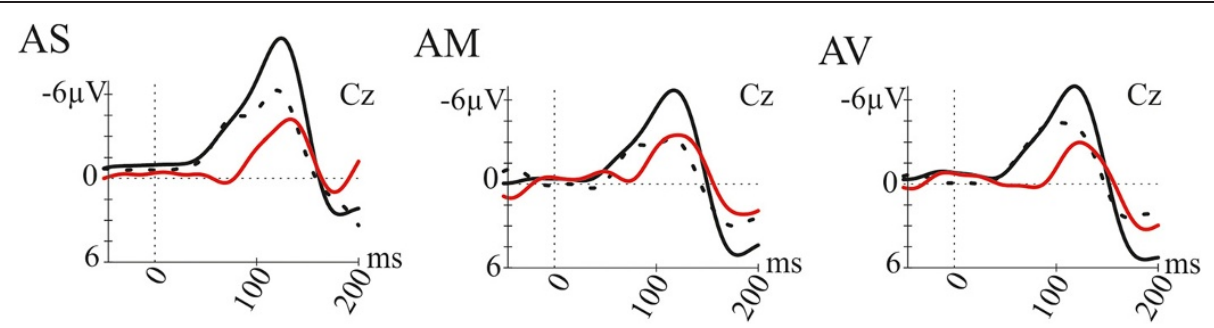

Legend:

$$
\begin{aligned}
& \text { - A } \\
& \text { - MA difference A minus MA }
\end{aligned}
$$

Figure 2 Illustration of the self-initiation effect for single attention conditions. Grand-average ERPs at Cz elicited by externally-initiated sounds (black solid line), self-initiated sounds (black dotted line) and the difference waves (externally-initiated minus self-initiated, red line), separately for the single attention conditions Attention Sounds (AS), Attention Motor (AM) and Attention Visual (AV).

when attending the sounds and when not attending the sounds as well as the attention effect (attended minus unattended) for the mean of self-initiated and externallyinitiated sounds. Furthermore, voltage maps and SCDs show the corresponding distribution over the scalp of the attention effect in all three N1 time windows (Figure 4B). The analysis for all N1 time windows revealed a main effect of Attention (N1b time window: $F(2,24)=32.45 ; p<$ .001 ; N1a time window: $F(2,24)=10.57 ; p=.001 ; \mathrm{N} 1 \mathrm{c}$ time window: $F(2,24)=38.39 ; p<.001)$. Pairwise comparison indicated higher activity for attending the sounds compared to not attending the sounds (N1b time window: $t(12)=-7.87 ; p<.001 ; \mathrm{N} 1 \mathrm{a}$ time window: $t(12)=-4.89$; $p<.001$; N1c time window: $t(12)=-8.28 ; p<.001)$. There was also a significant interaction of Attention $\times$ Laterality for the N1b time window $[F(8,96)=9.65 ; p<.001]$ and the N1a time window $[F(8,96)=4.82 ; p<.01]$. Pairwise comparisons for the N1b time window showed higher amplitudes

(A)

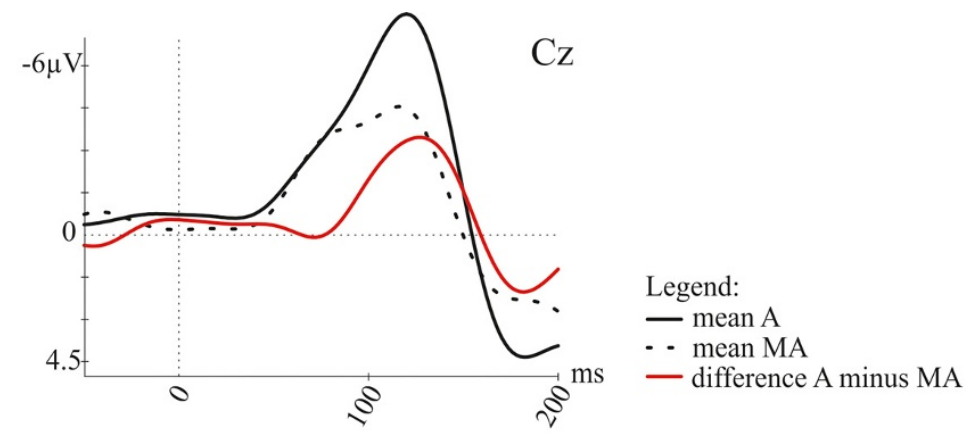

(B)
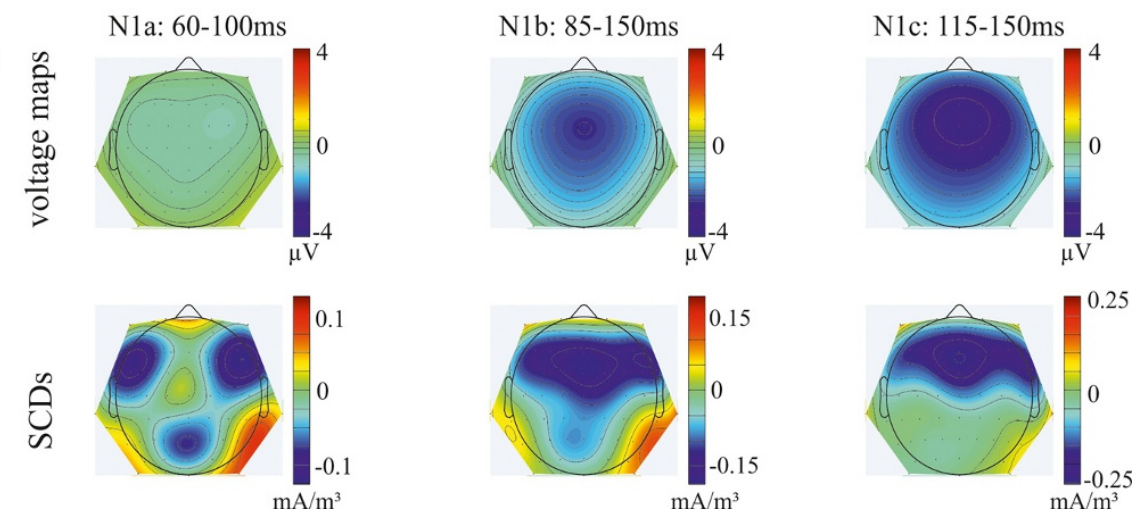

Figure 3 Illustration of the mean self-initiation effect. (A) Grand-average ERPs (mean of all attention conditions $A S, A M, A M$ at $C z$ elicited by externally-initiated sounds (black solid line), self-initiated sounds (black dotted line), as well as the difference wave (externally-initiated minus selfinitiated, red line). (B) Voltage maps and scalp current densities (SCDs) of the difference wave during the latency ranges of the N1a (60-100 ms), N1b (85-150 ms) and N1c (115-150 ms) time window. 
(A)

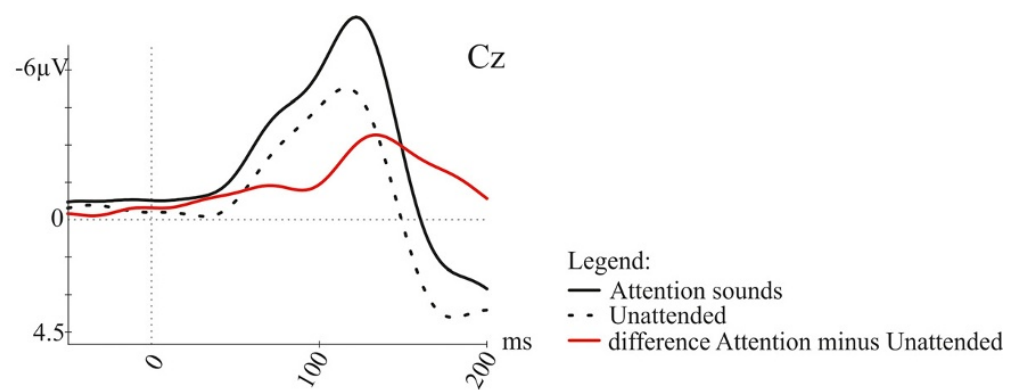

(B)
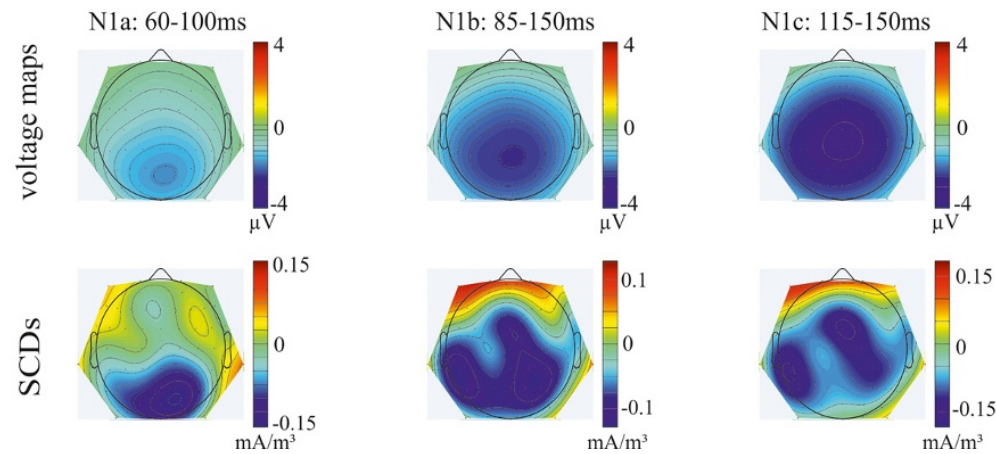

Figure 4 Illustration of the attention sound effect. (A) Grand-average ERPs (mean of self-initiated and externally-initiated sounds) at Cz elicited by attending the sounds (black solid line), not attending the sounds (black dotted line), as well as the difference wave (attended minus unattended, red line). (B) Voltage maps and scalp current densities (SCDs) of the difference wave during the latency ranges of the N1a (60-100 ms), N1b (85-150 ms) and N1c (115-150 ms) time window.

for attended compared to unattended sounds for all laterality levels [far left $(t(12)=-6.29 ; p<.001)$, left $(t(12)=-8.01 ; p<.001)$, midline $(t(12)=-8.90 ; p<.001)$, right $(t(12)=-7.50 ; p<.001)$, far right $(t(12)=-4.72$; $p<.001)$ ]. For the N1a time window the post-hoc analysis indicated higher amplitudes for attended compared to unattended sounds for all laterality levels except the far right (F8, T8, P8) level [far left $(t(12)=-3.23 ; p<.05)$, left $(t(12)=-5.03 ; p<.001)$, midline $(t(12)=-6.16 ; p<.001)$, right $(t(12)=-6.14 ; p<.001)$, far right $(t(12)=-2.54$; $p=.130)]$. For both time windows the attention effect shows a more parietal distribution (see Figure 4B, upper panel) compared to the self-initiation effect (see Figure 3B, upper panel).

The SCD distribution reveals that the putative sources of this attention effect are located in more parietal areas compared to the self-initiation effect (see Figure 3B, lower panel). However, the distribution for the N1b time window shows a more widespread activity than the distribution of the N1a time window (see Figure 4B, lower panel). Furthermore, there was a significant interaction of Attention and Anterior-Posterior for the N1b $[F(4,48)=17.83 ; p$ $<.001]$ and the N1a $[F(4,48)=6.37 ; p<.01]$ time window. Pairwise comparisons revealed higher activity for attended compared to unattended sounds for all levels of both time windows [N1b time window: frontal $(t(12)=-5.83 ; p<$ $.001)$, central $(t(12)=-7.86 ; p<.001)$, parietal $(t(12)=$ $-8.72 ; p<.001)$; N1a time window: frontal $(t(12)=-2.91$; $p=.039)$, central $(t(12)=-4.37 ; p<.01)$, parietal $(t(12)=-5.91 ; p<.001)]$. Again, this attention effect shows a parietal distribution (see Figure 4B, upper panel) which is supported by a parietal pattern of activity in the SCDs (see Figure 4B, lower panel). For the N1c time window no such interactions were found. However, the analysis revealed an interaction of Attention $\times$ Laterality $\times$ Anterior-Posterior $[F(16,192)=2.53 ; p<.05]$ for this time window, indicating a parietal and left-lateralized distribution of the attention effect which shows a more anterior distribution than the $\mathrm{N} 1 \mathrm{~b}$ and the N1a time window (see Figure 4B, upper panel). This finding is also supported by the SCDs which point at a more central topography (see Figure 4B, lower panel). Finally, at the mastoids no main effect of Attention was found $[F(2,24)=1.03 ; p=.374]$.

\section{Discussion}

In the present study we investigated to which extent the N1-suppression effect for self-initiated sounds can be explained by a differential allocation of attention to selfinitiated and externally-initiated sounds. To overcome possible limitations of the traditional blocked design self-initiated sounds and externally-initiated sounds as well as the motor control were presented within the same block. The allocation of attention was manipulated block-wise in three different attention conditions ( $A S$, $A M, A V)$, so that attention was directed to the sounds or was directed away from the sounds towards the own 
motor behavior or the visual stimulation. Moreover, we compared effects of self-initiation with attention effects to determine whether the underlying neural processes affect the same or different structures.

Horvath and colleagues (2012) have proposed that that N1 suppression might possibly be caused by split attentional resources in active conditions compared to passive conditions of the traditional blocked design [8-14]. We found an attenuation of the auditory $\mathrm{N} 1$ for self-initiated compared to externally-initiated sounds that was independent from the allocation of attention. That is, the N1 suppression was the same, irrespective of whether attention was directed to the sounds, directed to the motor act or directed to the visual stimuli. Thus, the N1-suppression effect cannot be explained by attentional differences between self- and externallyinitiated sounds. In other words, sensory suppression to selfinitiated sounds cannot be explained by the fact that the motor act draws away attention from auditory processing. Our finding is consistent with a recent study reporting reduced N1 amplitude during self-vocalization using a selective attention task to assess the N1 component independent of the attention effect [28].

Similar to forward modeling effects in other species $[29,30]$, it has been argued that the N1-suppression effect is a very basic and automatic phenomenon [17]. Horvath and colleagues (2012) showed that the auditory input seems to be attenuated for a short period after the motor act, even if there is no contingency between button press and sound. It seems that the sensory processing during self-initiation of sounds is merely affected by the concurrent motor act [31]. Our finding that the neural processes underlying the N1 suppression are not modulated by attention strongly supports the view that they are rather automatic. In fact, the definition of an automatic (versus a controlled) process is that it does not interfere with attention [32,33].

As predicted, the allocation of attention to the sounds resulted in an increase of the auditory $\mathrm{N} 1$, as compared to the N1 elicited by the sounds when attention was directed to the button presses or to the visual stimuli. This finding is consistent with results from previous studies [19,25,34-36]. However, previous studies often obtained a more fronto-central distributed auditory attention effect $[34,37,38]$, whereas we obtained a more parietal distribution. Nevertheless, top-down controlled attention has been reported to involve temporo-parietal and superior parietal areas [39], which is consistent with the distribution of our attention effect.

Moreover, the comparison of the self-initiation effect and the attention effect revealed that partly separate N1 components [25] are affected. Whereas all N1 components (i.e. N1a, N1b, N1c) were modulated by attention, only the late part of the N1 (i.e. N1b, N1c) was suppressed by self-initiation. Thus, we conclude that the predictive modeling underlying the N1-suppression effect is not "only" attention in time $[20,27]$ but a mechanism that is separable from a mere attentional mechanism. In the present report, the frontocentral peak of the N1b did not coincide with the time of polarity reversal at the mastoids, which occurred slightly earlier. The N1b component is known to receive contributions from both the tangentially oriented, sensory-specific component and the unspecific component of the N1 [25]. Because the unspecific component occurs later in time, its contribution tends to delay the peak of the N1b on frontocentral leads [40]. Thus, the window of analysis chosen here around the peak of the N1b probably receives its largest contribution from the unspecific N1 component. There were no self-initiation effects at the mastoids on the polarity-inverted N1 deflection. This finding suggests that a large part of the N1-suppression effect may be due to the suppression of the unspecific N1 component rather than the attenuation of sensory responses in auditory cortex as stipulated from internal predictive models theory. Thus, it could be speculated that the N1-suppression effect as measured in most ERP studies may largely reflect the fact that selfinitiated sounds are less arousing compared to externallyinitiated sounds. However, the lack of N1 suppression on the mastoids and on fronto-central electrodes at the time of polarity reversal at the mastoids in the present experiment does not necessarily imply that sensory responses are not attenuated by self-initiation in auditory cortex at all. Indeed, previous MEG studies, which specifically measure the activity of tangentially oriented sources on auditory cortex, have found N1 suppression for self-initiated sounds $[8,12,17]$.

\section{Conclusions}

We could show that the N1 suppression was equally large and of equal distribution when subjects directed their attention towards the sound and when the directed their attention away from the sounds, towards the button presses or the visual stimuli. Thus, the self-initiation effect can hardly be explained by the differential amount of attention devoted to self- and externally-triggered sounds. Instead, the present results support the notion that N1 suppression for self-initiated sounds seems to reflect the activity of an internal predictive mechanism. Whereas the effects of voluntary attention affect all N1 components, the self-initiation effect seems to be confined to the N1b and N1c components. The present mixed design provides a useful tool to measure genuine self-initiation effects.

\section{Methods}

\section{Participants}

Fifteen healthy volunteers (7 male, 1 left-handed) participated in the experiment. Two male participants had to be excluded from the analysis due to low signal-to-noise ratio. Mean age of the remaining thirteen participants 
was 22.92 years (range: 19 to 29 years). All participants reported normal hearing and normal or corrected-tonormal vision. None were taking any medication affecting the central nervous system. All participants received either course credit or payment for their participation. The experiment was undertaken with the understanding and written consent of each subject. The experimental protocol conformed to the Declaration of Helsinki and the ethics guidelines of the German Association of Psychology (ethics board of the Deutsche Gesellschaft für Psychologie, DGPs: http://www.dgps.de/dgps/aufgaben/ ethikrl2004.pdf) and did thus not require any additional ethics approval.

\section{Experimental conditions}

Participants were asked to fixate on a grey cross constantly displayed on the center of a black screen. Small extensions of the fixation cross (from a visual angle of $0.69^{\circ}$ to $0.74^{\circ}$ with a distance to the monitor of $100 \mathrm{~cm}$ ) were presented for $80 \mathrm{~ms}$ duration using a variable stimulus onset asynchrony (SOA) of 5-15 s. These extended fixation crosses were not predictable for the participants. Using a mixed experimental design selfinitiated and externally-initiated sounds were presented in the same block (Figure 5). Participants were instructed to press a button with their left or right thumb (depending on handedness) with self-paced intervals of 5-8 s (mean: 6.5 s). In $50 \%$ of the trials button presses initiated a $50 \mathrm{~ms}$ sine tone of $1000 \mathrm{~Hz}$ (including 10-ms rise and 10-ms fall times) which was presented immediately after the button press through headphones (Sennheiser HD 25-1) (motor-auditory condition in the blocked design, MA). The intensity of the sounds was adjusted to a comfortable loudness by the participant with soft foam earplugs inserted to attenuate any other sounds. In the remaining $50 \%$ of the trials button presses were not followed by any sound (motor-only condition in the blocked design, M). For the participants it was not predictable whether the button press would initiate a sound or not. Additionally, externally-initiated sounds (with the same physical parameters as the self-initiated sounds) were presented randomly between button presses (auditory-only condition in the blocked design, A). Externally-initiated sounds were unpredictable in their occurrence. The SOA between two externally-initiated sounds ranged randomly between 5-8 s. All sounds were generated with MATLAB (http://www.mathworks.com). To avoid a possible overlap with preceding self-initiated sounds, externally-initiated sounds were always presented at least $1 \mathrm{~s}$ after the occurrence of a button press. When the SOA between a preceding externally-initiated sound and a button press (initiating a sound or not) was smaller than $1 \mathrm{~s}$ both trials were excluded, but the respective number of trials were added at the end of the block to avoid loss of data. In addition to the self-initiation task the allocation of attention was manipulated block-wise. Three attention conditions were included (Attention Sound, Attention Motor, Attention Visual). In the Attention Sound (AS) condition participants were instructed to count all sounds they could hear, including self-initiated and externally-initiated ones. In the Attention Motor (AM) condition participants counted all button presses they made. In the Attention Visual ( $A V$ ) condition they were asked to count all extended fixation crosses they saw on the screen. Thus, less attention should be directed to the

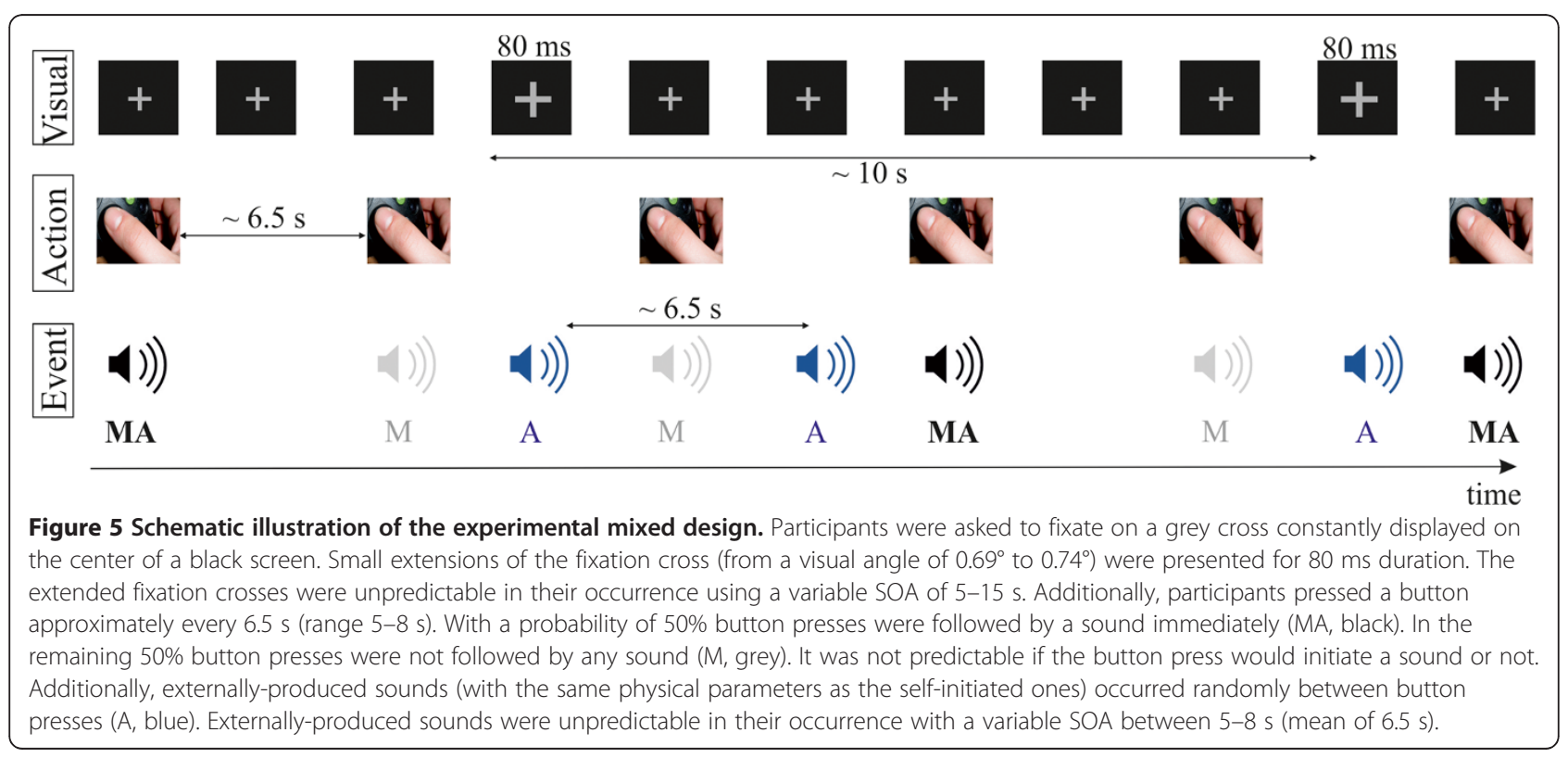


sounds when participants attend to the motor act or to the visual stimuli than when they attend to the sounds.

\section{Experimental procedure}

During EEG recordings, participants were seated in a sound-attenuated and electrically shielded chamber. Auditory stimulation was run via MATLAB using the Cogent2000 toolbox (http://www.vislab.ucl.ac.uk/cogent_2000. php). Participants were instructed to press the button once every 5-8 s (mean: $6.5 \mathrm{~s}$ ). They were informed that a button press would be followed by a sound or silence. Participants were informed about the occurrence of the externallyinitiated sounds. However, they were not provided with further information about them. To get used to the selfinitiation task participants received several training blocks before the experiment. In these training blocks visual feedback of the button press SOA was given after each button press. In the main experiment visual feedback about the mean button press interval and the responses that were too slow or too fast were only shown at the end of each block. To avoid data loss, a block was repeated whenever participants pressed the button more than 5 times too slow or too fast within one block. In addition to the self-initiation task, participants had to count either all the sounds they could hear $(A S)$, all the button presses they made $(A M)$ or all the extended fixation crosses they saw $(A V)$. Participants were always informed before the beginning of each block about the respective task. After each block they reported the number of counted events. To make sure participants attended to the particular events effectively the block was repeated whenever they miscounted more than $+/-2$. Meta-blocks, including all three attention conditions, were repeated eight times. Thus, the EEG experiment consisted of twenty-four experimental blocks. In the meta-blocks the attention conditions $(A S, A M, A V)$ were pseudo-randomized.

Each block consisted on average of twelve (range: ten to fourteen) self-initiated sounds (MA) and silent button presses $(\mathrm{M})$, respectively. This variation was included to make the counting task less predictable for the participants. A comparable number of externally-initiated sounds (A) was presented depending on the mean SOA of the self-paced button presses. In total a mean of 96 trials were analysed for each event (MA, A, M) for each attention condition ( $A S, A M, A V)$, respectively.

\section{Data recording and analysis}

EEG activity was recorded continuously with $\mathrm{Ag} / \mathrm{AgCl}$ electrodes from 60 standard locations (Fp1, Fp2, AF3, AFz, AF4, F7, F5, F3, F1, Fz, F2, F4, F8, FT7, FC3, FC1, $\mathrm{FCz}, \mathrm{FC} 2, \mathrm{FC} 4, \mathrm{FC} 6, \mathrm{FT} 8, \mathrm{~T} 7, \mathrm{C} 5, \mathrm{C} 3, \mathrm{C} 1, \mathrm{Cz}, \mathrm{C} 2, \mathrm{C} 4$, C6, T8, TP7, CP5, CP3, CP1, CPz, CP2, CP4, CP6, TP8, P7, P5, P3, P1, Pz, P2, P4, P6, P8, PO9, PO7, PO3, POz, $\mathrm{PO} 4, \mathrm{PO} 8, \mathrm{PO} 10, \mathrm{O} 1, \mathrm{Oz}, \mathrm{O} 2)$ according to the international 10-20 electrode system [41] including the left and right mastoid (M1, M2). An additional electrode was placed at the tip of the nose (serving as offline reference). EOG was measured using the setup described by [42] with one electrode at nasion and two electrodes at the outer canthi. EEG signals were sampled at $500 \mathrm{~Hz}$.

Automatic eye movement correction was applied on the data according to the procedure described in [42], preceded by a 1 to $100 \mathrm{~Hz}$ offline band-pass filter. After EOG artifact correction, data were filtered with a 1-25 Hz band-pass filter (kaiser-window, ripple: 0.017, length: 5653 points). For each trial, an epoch of $600 \mathrm{~ms}$ duration including a $200 \mathrm{~ms}$ pre-stimulus baseline was extracted from the continuous EEG record. Epochs with amplitude changes exceeding $75 \mu \mathrm{V}$ on any channel were rejected from further analysis. ERPs were averaged time-locked to stimulus onset separately for each event type, attention condition and participant. Button press errors (inter-press interval $<5000 \mathrm{~ms}$ or $>8000 \mathrm{~ms}$ ) were removed from the EEG analysis.

To correct for motor activity present in responses to self-initiated sounds, the ERPs elicited by button presses followed by no sound were subtracted from the ERPs elicited to the self-initiated sounds. This motor-responsecorrected ERP was then compared with the ERP of the externally-initiated sounds. In all figures and analysis, ERPs elicited by the self-initiated sounds were corrected this way. This approach has become an appropriate procedure in previous research (presenting MA and M conditions in separate blocks) to measure auditory processing activity in the presence of motor-related activity. However, presenting MA and M conditions introduces a possible confound, namely that it cannot be completely ruled out that non-motor responses, e.g. responses related to temporal expectations of the sound, might also be eliminated subtracting the ERPs elicited by button presses followed by no sound from the ERPs elicited to the selfinitiated sounds. However, as the N1-suppression effect observed in the present study was virtually identical to the one reported in previous studies using no mixed design suggests that the suppression effects are not an artefact of the subtraction method of the mixed design.

Because of the multiple components with separate and potentially overlapping latencies underlying the $\mathrm{N} 1$ [25] we investigated three separate intervals in the N1 latency range which fit to the peaks N1a, N1b and N1c that have been described in the literature before $[25,26,40,43]$. Intervals for the N1a and N1c peaks were defined to encompass the first and second peak of the N1 at temporal electrodes. The interval for the N1b peak was defined to encompass the broader N1 peak at central and frontal electrodes. Thus, ERP effects were investigated around the grandaverage peaks in the latency range of $85-150 \mathrm{~ms}$ (N1b time window), 60-100 ms (N1a time window) and 115-150 ms (N1c time window) after stimulus onset (see Figure 1). 
ERP amplitudes were calculated from the individual averages as the mean amplitude within these specified analysis time windows. A repeated measurement analysis of variance (ANOVA) with the factors Attention (AS, AM, AV), Production (self-initiated vs. externally-initiated), Laterality (far left: F7, T7, P7; left: F3, C3, P3; midline: Fz, Cz, Pz; right: F4, C4, P4; far right: F8, T8, P8) and Anterior-Posterior (frontal: F7, F3, Fz, F4, F8; central: T7, C3, Cz, C4, T8; parietal: P7, P3, Pz, P4, P8) was computed for each N1 time window, on the mean amplitudes of the electrodes F7, T7, P7, F3, C3, P3, Fz, Cz, Pz, F4, C4, P4, F8, T8, P8. Moreover, in order to identify the sensory specific N1 component generated in auditory cortex, a further repeated measurement ANOVA with the factors Attention $\times$ Production was calculated for the mastoid signals in the latency range of $70-110 \mathrm{~ms}$, since the generator for this component has a tangential orientation and results in N1 responses which are negative over frontocentral locations but are also recorded with inverted polarity on the mastoids.

For studying the scalp topographies in the interesting latency ranges, ERP voltage distributions were transformed into scalp current density (SCD) distributions, computing the second spatial derivative of the interpolated potential distribution $[44,45]$. The maximum degree of the Legendre polynomials was chosen to be 50 , and the order of splines (m) was set to 4. A smoothing parameter lambda of $10^{-4}$ was applied. For behavioural data a one-way repeated ANOVA with the factor Attention was computed to compare inter-press time intervals, total number of button presses and timing errors for the self-initiation task between the attention conditions $(A S, A M, A V)$. Furthermore, the counting rates of the attention task for all attention conditions were compared. The counting rates represent the total number of correctly counted events in relation to the total number of actual events of each attention condition. Greenhouse-Geisser correction was applied where appropriate. Additional pairwise comparisons ( $\mathrm{p}$-value alphaadjusted using the Bonferroni correction) were conducted when appropriate to clarify the origin of significant effects. Only interactions that are relevant for the addressed question are reported.

\section{Endnotes}

${ }^{\mathrm{a}}$ As the recording of neural responses to motor activity without sounds in separate experimental blocks and subtracting these responses from the motor responses of the active condition could lead to biased estimates of sensory processing $[15,17]$, we used a variant of the mixed N1 suppression paradigm, in which $50 \%$ of the button presses trigger a sound while the other $50 \%$ do not. With this, the representation of the motor command (efference copy) should be fully eliminated.

\section{Additional files}

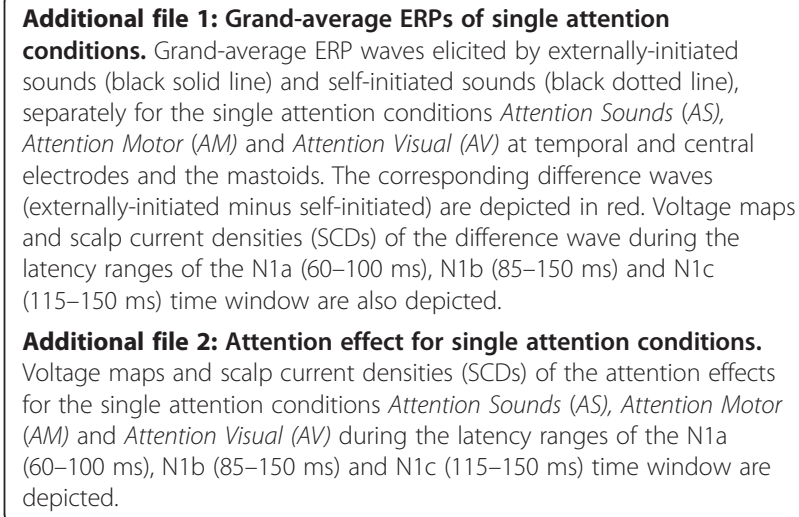

Authors' contributions

JT, IS, KS, and ES designed the study. JT, IS and KS programmed the task. JT acquired the data. JT, IS and KS performed the data analysis. All authors participated the data evaluation and interpretation and in writing the manuscript, and have approved the final version of the manuscript.

\section{Acknowledgements}

This work was supported by a Reinhart-Koselleck grant of the German Research Foundation (Deutsche Forschungsgemeinschaft, DFG, Project SCHR 375/20-1).

It was realized using Cogent 2000 developed by the Cogent 2000 team at the FIL and the ICN and Cogent Graphics developed by John Romaya at the LON at the Wellcome Department of Imaging Neuroscience. The authors wish to thank Andreas Widmann for providing the scripts for eye movement correction and Alexandra Bendixen for providing the scripts for intensity measurement.

Received: 17 August 2012 Accepted: 29 December 2012 Published: 3 January 2013

\section{References}

1. Wolpert DM, Ghaharamani Z, Jorden MI: An internal model for sensorimotor integration. Science 1995, 269:1180-1182.

2. Wolpert DM, Ghaharamani Z: Computational principles of movement neuroscience. Nat Neurosci 2000, 3:1212-1217.

3. Wolpert DM, Flanagan JR: Motor prediction. Curr Biol 2001, 11:R729-R732.

4. Von Holst E, Mittelstaedt H: Das Reafferenzprinzip. Naturwissenschaften 1950, 37:464-467.

5. Sperry RW: Neural basis of the spontaneous optokinetic response produced by visual inversion. J Comp Physiol Psychol 1950, 43:482-489.

6. Blakemore SJ, Wolpert DM, Frith C: Why can't you tickle yourself? Neuroreport 2000, 11:R11-R16.

7. Weiskrantz L, Elliott J, Darlington C: Preliminary observations on tickling oneself. Nature 1971, 230:598-599.

8. Aliu SO, Houde JF, Nagarajan SS: Motor-induced suppression of the auditory cortex. J Cogn Neurosci 2009, 21:791-802.

9. Baess P, Jacobsen T, Schroger E: Suppression of the auditory N1 event-related potential component with unpredictable self-initiated tones: evidence for internal forward models with dynamic stimulation. Int J Psychophysiol 2008, 70(2):137-143.

10. Ford JM, Gray M, Faustman WO, Roach BJ, Manthalon DH: Dissecting corollary discharge dysfunction in schizophrenia. Psychophysiology 2007, 44:522-529.

11. Knolle F, Schröger E, Baess P, Kotz SA: The cerebellum generates motor-to-auditory predictions: ERP lesion evidence. J Cogn Neurosci 2012, 24:698-706.

12. Martikainen MH, Kaneko K, Hari R: Suppressed responses to self-triggered sounds in the human auditory cortex. Cereb Cortex 2005, 15:299-302.

13. McCarthy $G$, Donchin $E:$ The effects of temporal and event uncertainty in determining the waveforms of the auditory event related potential (ERP). Psychophysiology 1976, 13:581-590. 
14. Schafer EW, Marcus MM: Self-stimulation alters human sensory brain responses. Science 1973, 181:175-177.

15. Hughes G, Desantis A, Waszak F: Mechanisms of intentional binding and sensory attenuation: the role of temporal prediction, temporal control, identity prediction, and motor prediction. Psychol Bull 2012. doi:10.1037/ a0028566. Advance online publication.

16. Baess P, Horvath J, Jacobsen T, Schröger E: Selective suppression of self-initiated sounds in an auditory stream: an ERP study. Psychophysiology 2011, 48:1276-1283.

17. Horvath J, Maess B, Baess P, Toth A: Action-sound coincidences suppress evoked responses of the human auditory cortex in EEG and MEG. J Cogn Neurosci 2012, 24:1919-1931.

18. Hillyard SA, Hink RF, Schwent VL, Picton TW: Electrical signs of selective attention in the human brain. Science 1973, 182:177-180.

19. Hillyard SA: Selective auditory attention and early event-related potentials: a rejoinder. Can J Psychol 1981, 35:159-174.

20. Nobre AC: Orienting attention to instants in time. Neuropsychologia 2010 39:1317-1328.

21. Alho K, Vorobyev VA: Brain activity during selective listening to natural speech. Front Biosci 2007, 12:3167-3176.

22. Horvath J, Winkler I: Distraction in a continuous-stimulation detection task. Biol Psychol 2010, 83:229-238.

23. Logan GD: Attention in character-classification tasks: evidence for the automaticity of component stages. J Exp Psychol 1978, 107:32-63.

24. Logan GD: On the use of a concurrent memory load to measure attention and automaticity. J Exp Psychol 1979, 5:189-207.

25. Näätänen $\mathrm{R}$, Picton TW: The $\mathrm{N} 1$ wave of the human electric and magnetic response to sound: a review and an analysis of the component structure. Psychophysiology 1987, 24:375-425.

26. Woods DL: The component structure of the N1 wave of the human auditory evoked potential. Electroencephalogr Clin Neurophysiol 1995, 44:102-109.

27. Lange K: The reduced $\mathrm{N} 1$ to self-generated tones: an effect of temporal predictability? Psychophysiology 2011, 48:1-8.

28. Kudo N, Nakagome K, Kasai K, Araki T, Fukuda M, Kato N, Iwanami A: Effects of corollary discharge on event-related potentials during selective attention task in healthy men and women. Neurosci Res 2004 48:59-64.

29. Eliades SJ, Wang $X$ : Sensory-motor interaction in the primate auditory cortex during self-initiated vocalizations. J Neurophysiol 2003, 89:2194-2207.

30. Müller-Preuss P, Ploog D: Inhibition of auditory cortical neurons during phonation. Brain Res 1981, 215:61-76.

31. Makeig S, Müller M, Rockstroh B: Effects of voluntary movements on early auditory brain responses. Exp Brain Res 1996, 110:487-492.

32. Kahneman D, Treisman A: Changing views of attention and automaticity. In Varieties of attention. Edited by Parasuraman D. New York: Academic Press; 1984:29-61.

33. Hackley SA: An evaluation of the automaticity of sensory processing using event-related potentials and brain-stem reflexes. Psychophysiology 1993, 5:415-428

34. Alho K, Woods DL, Algazi A: Processing of auditory stimuli during auditory and visual attention as revealed by event-related potentials. Psychophysiology 1994, 31:469-479.

35. Giard MH, Perrin J, Peronnet F: Several attention-related wave forms in auditory areas: a topographic study. Electroencephalogr Clin Neurophysiol 1988, 69:371-384.

36. Woldorff MG, Hillyard SA: Modulation of early auditory processing during selective listening to rapidly presented tones. Electroencephalogra Clin Neurophysiol 1991, 79:170-191.

37. Talsma D, Kok A: Nonspatial intermodal selective attention is mediated by sensory brain areas: evidence from event-related potentials. Psychophysiology 2001, 38:736-751.

38. Talsma D, Kok A: Intermodal spatial attention differs between vision and audition: an event-related potential analysis. Psychophysiology 2002, 39:689-706

39. Salmi J, Rinne T, Koistinen S, Salonen O, Alho K: Brain networks of bottomup triggered and top-down controlled shifting of auditory attention. Brain Res 2009, 1286:155-164.
40. Budd TW, Barry RJ, Gordon E, Rennie C, Michie PT: Decrement of the N1 auditory event-related potential with stimulus repetition: habituation vs. refractoriness. Int J Psychophysiol 1998, 31:51-68.

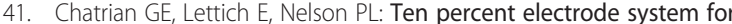
topographic studies of spontaneous and evoked EEG activities. Am J Electroencephalogr Technol 1985, 25:83-92.

42. Schlögl A, Keinrath C, Zimmermann D, Scherer R, Leeb R, Pfurtscheller G: A fully automated correction method of EOG artifacts in EEG recordings. Clin Psychol 2007, 118:98-104

43. Wolpaw JR, Pentry JK: A temporal component of the auditory evoked response. Electroencephalogr Clin Neurophysiol 1975, 39:609-620.

44. Perrin F, Pernier J, Bertrand O, Echallier JF: Spherical splines for scalp potential and current density mapping. Electroencephalogr Clin Neurophysiol 1989, 72:184-187.

45. Perrin F, Pernier J, Bertrand O, Echallier JF: Corrigendum. Electroencephalogr Clin Neurophysiol 1990, 76:565.

doi:10.1186/1471-2202-14-2

Cite this article as: Timm et al.: The N1-suppression effect for selfinitiated sounds is independent of attention. BMC Neuroscience 2013 $14: 2$

\section{Submit your next manuscript to BioMed Central and take full advantage of:}

- Convenient online submission

- Thorough peer review

- No space constraints or color figure charges

- Immediate publication on acceptance

- Inclusion in PubMed, CAS, Scopus and Google Scholar

- Research which is freely available for redistribution
C Biomed Central 